\section{$\underset{\substack{\text { hommes } \\ \text { \& migrations }}}{ }$}

\section{Hommes \& migrations}

Revue française de référence sur les dynamiques

migratoires

\section{$1322 \mid 2018$}

Exposer les migrations

\title{
Exposer des mémoires de migrations
}

Retour sur une collecte participative

Helene Bertheleu, Véronique Dassié, Guillaume Etienne et Julie Garnier

\section{Q OpenEdition \\ 1 Journals}

Édition électronique

URL : https://journals.openedition.org/hommesmigrations/6687

DOI : 10.4000/hommesmigrations.6687

ISSN : 2262-3353

Éditeur

Musée national de l'histoire de l'immigration

Édition imprimée

Date de publication : 1 juillet 2018

Pagination : 113-122

ISBN : 978-2-919040-42-1

ISSN : 1142-852X

Référence électronique

Helene Bertheleu, Véronique Dassié, Guillaume Etienne et Julie Garnier, «Exposer des mémoires de migrations », Hommes \& migrations [En ligne], 1322 | 2018, mis en ligne le 01 juillet 2020, consulté le 21 janvier 2022. URL : http://journals.openedition.org/hommesmigrations/6687 ; DOI : https://doi.org/ 10.4000/hommesmigrations.6687 


\title{
EXPOSER DES MÉMOIRES DE MIGRATIONS RETOUR SUR UNE COLLECTE PARTICIPATIVE
}

Par HÉLÈNE BERTHELEU, sociologue, maître de conférences, université de Tours, CNRS-Citeres, VÉRONIQUE DASSIÉ, anthropologue, MSH Aix-Marseille, CNRS-Idemec, GUILLAUME ÉTIENNE, anthropologue, université de Tours, CNRS-Citeres, et JULIE GARNIER, sociologue, maître de conférences, université de Tours, CNRS-Citeres.

\author{
Les concepteurs d'expositions traitant des thématiques \\ migratoires privilégient actuellement des démarches \\ participatives en lien avec le monde de la recherche et le milieu \\ associatif. L'analyse de la collaboration entre ces différents \\ acteurs aux positions sociales et professionnelles différentes, \\ autour d'un projet d'exposition porté par une association, \\ conduit à questionner le processus même de fabrication \\ du patrimoine. Ainsi, les procédés d'enquête et de collecte \\ mobilisés révèlent tout autant les difficultés inhérentes \\ à la patrimonialisation que la formidable capacité \\ d'une exposition à créer du lien social, depuis sa conception \\ jusqu'à sa présentation au public.
}

Depuis les années 1990, les travaux sur les patrimonialisations ${ }^{1}$ ont remis en question les visions essentialistes du patrimoine, montrant que ce dernier n'existe pas en soi mais qu'il est le résultat d'un processus qui met en jeu des acteurs aux compétences et statuts variés. Que ce soit au sein des administrations du patrimoine ou des associations à vocation culturelle ou sociale, ce «basculement d'un patrimoine d'objets (un "trésor" déjà amoncelé) en patrimoine en projet (tout ce qu'en principe il faut ou faudrait conserver et sauvegarder) $)^{2}$ " a conduit à en faire le lieu de passions ${ }^{3}$, de réappropriations mémorielles ${ }^{4}$, voire de revendications ${ }^{5}$, de déplorations et de résistances ${ }^{6}$ ou de conflits ${ }^{7}$, autrement dit un instrument politique ${ }^{8}$ qui pose à nouveaux frais la question de l'engagement patrimonial.

Dans la continuité de ces travaux, nous interrogerons l'irruption de ces nouveaux acteurs dans la fabrique patrimoniale ${ }^{9}$, le sens de leur engagement et ses effets, à partir d'une recherche menée entre 
2014 et $2017^{10}$ sur la réalisation d'une exposition sur les migrations dans laquelle nous avons été impliqués ${ }^{11}$. Cette recherche avait pour objectif d'analyser les conditions de mise en patrimoine des migrations, non pas à travers l'observation d'un protocole d'enquête-collecte tel qu'il aurait pu être mis en place par un musée, mais à travers l'analyse d'une situation mettant en présence une diversité d'acteurs, autour d'un projet d'exposition porté par une association ${ }^{12}$. Dans ce contexte atypique, qui fait patrimoine dans des démarches qui se veulent partagées? L'appel à participation, lancé au-delà des publics d'un musée, permet-il de faire entrer dans le cercle des artisans de l'exposition des personnes directement concernées par la migration?

L’appel à participation,

lancé au-delà des publics

d'un musée, permet-il

de faire entrer dans le cercle des artisans de l'exposition

des personnes

directement concernées par la migration ?

Notons d'emblée que notre recherche ne porte pas sur l'institution patrimoniale en tant que dispositif public concerné par une telle patrimonialisation, mais sur la manière dont les migrations peuvent, à un moment donné, rencontrer la cause patrimoniale. En 2013, nous avons en effet été sollicités en tant que collectif de chercheurs spécialistes des migrations et du patrimoine par l'association Mémoires plurielles ${ }^{13}$ pour mettre en place une exposition sur le thème des migrations en région Centre. Cela permettrait, d'une part, de valoriser une précédente enquête sur les mémoires des migrations ${ }^{14}$ et d'élargir les destinataires des connaissances établies. Elle nous encourageait, d'autre part, à expérimenter un mode de recherche collaboratif en nous associant à une institution patrimoniale (le Musée d'histoire et d'archéologie d'Orléans) et à une association de valorisation des mémoires des migrations. Elle nous permettait, enfin, d'explorer la mise en œuvre d'une "démocratie patrimoniale $e^{15}$ », la « production de significations sous le patronage du patrimoine [qui] concerne et engage une pluralité d'acteurs ${ }^{16}$ ", censée favoriser l'«émancipation de l'activité patrimoniale des cadres administratifs et scientifiques ${ }^{17} \gg$ dans la continuité des réflexions conduites entre 2012 et 2015 avec le groupement d'intérêt scientifique Institutions patrimoniales et pratiques interculturelles (GIS Ipapic) ${ }^{18}$.

Dans ce qui suit, nous présentons d'abord la manière dont cette exposition s'est construite et la dynamique réticulaire qu'elle a suscitée, puis nous questionnerons notre propre engagement en tant que collectif de chercheurs dans ce projet. Nous prendrons appui pour cela sur les résultats d'une enquête qui a consisté à ethnographier la participation ${ }^{19}$ pendant toute la durée de préparation de l'exposition ${ }^{20}$. L'analyse de cette situation de patrimonialisation apporte, nous le verrons, un éclairage intéressant sur le passage de la fabrique du patrimoine par l'institution à celle d'une fabrique sociale ${ }^{21}$ portée par ou avec des groupes sociaux. Elle amène à considérer la préparation de l'exposition comme un espace d'«engagement

10. Cette recherche, "L'exposition et ses effets. Territoires de migrations, démocratie patrimoniale et pratiques scientifiques renouvelées ", conduite par Véronique Dassié, Julie Garnier et Guillaume Étienne sous la direction d’Hélène Bertheleu, a bénéficié du soutien du ministère de la Culture et de la communication dans le cadre de l'appel à projet de recherche "Pratiques interculturelles dans les processus de patrimonialisation ", Iancé à l'initiative du GIS Ipapic en 2014. 11. L'exposition Histoires de migrations a été présente au Musée d'histoire et d'archéologie d'Orléans de mars à juillet 2017. 12. Cette recherche a donné lieu à la publication en 2015 d'un rapport et d'un catalogue d'exposition. Voir Guillaume Étienne (dir.), Histoires de migrations. Intimités et espaces publics, Tours, Presses universitaires François-Rabelais, 2017. 13. Association loi 1901 déclarée en novembre 2008 , qui a pour vocation de rassembler un réseau d'acteurs associatifs et institutionnels (travailleurs sociaux, chercheurs, enseignants, archivistes, artistes, médiateurs culturels, associations, etc.) susceptibles de valoriser les mémoires des migrations dans la région Centre. Elle bénéficie des soutiens de la Région Centre, de la DRAC Centre, et de la DRJSCS Cohésion sociale. Site Internet collaboratif : http://www.memoiresplurielles.fr. 14. Hélène Bertheleu (dir.), Au nom de la mémoire. Le patrimoine des migrations en région Centre, Tours, Presses universitaires François-Rabelais, 2014. 15. Jean-Louis Tornatore et Michel Rautenberg organisaient en 2012, dans le cadre du XIXe congrès de I'Association internationale des sociologues de langue française (AISLF) à Rabat (au Maroc), un groupe de travail dédié à cette question. 16. Jean-Louis Tornatore (dir.), L'invention de la Lorraine industrielle, op. cit. 17. Jean-Louis Tornatore, "Patrimoine vivant et contributions citoyennes. Penser le patrimoine "devant" l'Anthropocène ", in In Situ, n 33, 2017. 18. http://www.ipapic.eu/ 19. Daniel Cefaï, "Introduction. L'engagement ethnographique ", in L'engagement ethnographique, Paris, éd. de l’EHESS, 2010, pp. 7-21. 20. L'ensemble des réunions et des échanges ont été enregistrés de façon à pouvoir analyser, a posteriori, l'ensemble du processus de patrimonialisation et ses étapes. 21. Michel Rautenberg, La rupture patrimoniale, op. cit. ; Chiara Bortolotto, Le patrimoine culturel immatériel. Enjeux d'une nouvelle catégorie, Paris, éd. de la MSH, 2011. 
patrimonial ${ }^{22}$ ", où une multiplicité d'initiatives, expertes ou profanes, institutionnelles ou non peuvent converger. Elle permet, par ailleurs, d'interroger les conditions de patrimonialisation d'une mémoire aux côtés d'une diversité d'acteurs impliqués et concernés par la démarche.

\section{Genèse de la démarche}

Le projet d'exposition proposé par l'association régionale Mémoires plurielles est d'abord discuté avec les chercheurs et la conservatrice, directrice des musées d'Orléans. Un premier inventaire des ressources disponibles au musée et des éléments scientifiques accumulés par les chercheurs ${ }^{23}$ est dressé : aux yeux de la directrice du musée, cela suffit à défendre l'intérêt et le sérieux du projet d'exposition auprès des autorités culturelles locales. Toutefois, la décision de ne pas s'appuyer uniquement sur ces travaux scientifiques s'est rapidement imposée aux différents partenaires. À leurs yeux, associer des personnes " concernées » et «initiées ${ }^{24}$ " à la conception de l'exposition, permettrait de construire une narration «plus sensible » qui porterait plus efficacement le message de l'exposition.

Pour les chercheurs, cette condition est la garantie d'une démarche scientifique citoyenne et d'une restitution des contenus scientifiques à leurs informateurs. Pour l'association régionale, ce choix méthodologique est approprié, cohérent avec sa vocation à diffuser largement des connaissances locales sur les migrations, à établir des partenariats avec les acteurs les plus divers ${ }^{25}$, et à faire réfléchir par ce biais sur la question des discriminations, hier et aujourd'hui. Pour le musée, une telle implication est une manière d'élargir l'audience muséale au-delà de ses publics habituels. Aux yeux des partenaires financiers comme la région, la Direction régionale des affaires culturelles (DRAC), la direction régionale de la jeunesse, des sports et de la cohésion sociale (DRJSCS) et la ville, la dimension participative contribue à donner du crédit au projet, lui donnant une assise territoriale concrète, avec ses groupes de travail dans les quatre agglomérations de Bourges, Montargis, Orléans, et Tours ${ }^{26}$.

Un vaste appel à « participer tant à l'esprit du projet qu’à sa réalisation » est donc lancé lors d'une journée régionale d'échanges préalables organisée à Orléans d'abord, puis de façon plus ciblée dans les quatre agglomérations ${ }^{27}$. La vocation régionale du projet encourageait ce choix de La vocation régionale du projet encourageait ce choix de multiplier les collectifs de travail territorialisés. Pour les construire, chaque chercheur a mobilisé ses réseaux d'informateurs. multiplier les collectifs de travail territorialisés. Pour les construire, chaque chercheur a mobilisé ses réseaux d'informateurs. Chaque agglomération présentant des caractéristiques territoriales différentes, le projet embrassait ainsi une diversité de situations migratoires. Pôleth Wadbled, la chargée de mission de l'association Mémoires plurielles, a animé ces différents groupes de travail au côté des chercheurs. Dans le même

22. Jean-Louis Tornatore, "Patrimoine vivant et contributions citoyennes. Penser le patrimoine "devant" l'Anthropocène ", op. cit. 23. Sylvie Aprile, Hélène Bertheleu, Pierre Billion (dir.), Étrangers dans le berceau de la France ? L'immigration en région Centre du 19e siècle à nos jours, Tours, Presses universitaires François-Rabelais, 2013 ; Hélène Bertheleu (dir.), Au nom de la mémoire, op. cit. ; Hélène Bertheleu (dir.), Mémoires des migrations en France. Du patrimoine à la citoyenneté, Rennes, Presses universitaires de Rennes, 2016. 24. Cette distinction entre "concernés " et "initiés " est le fruit d'un premier travail où l'on avait repéré deux profils distincts de personnes mobilisées " au nom de la mémoire ". Les " concernés » sont ceux qui se sentent directement touchés par la question et deviennent des "entrepreneurs de mémoire ". Leur participation est à saisir "à la lumière de leur trajectoire et des appartenances sociales, culturelles, professionnelles et résidentielles souvent combinées " qui les caractérisent. Les " initiés " constituent le cercle plus large de ceux qui prennent le parti de "partager le concernement ». Voir E. Goffman, Stigmate. Les usages sociaux des handicaps, Paris, Les Éditions de Minuit, 1977, p. 43. Ils «épousent " en quelque sorte la cause, ici mémorielle et patrimoniale, des concernés. Ces initiés se mobilisent par loyauté, au nom de l'amitié ou parce que les liens tissés avec des migrants les ont amenés à vivre des expériences fortes, voire bouleversantes. Voir Hélène Bertheleu (dir.), Au nom de la mémoire, op. cit., p. 314. 25. Mémoires Plurielles collabore régulièrement avec l'université de Tours, des centres sociaux, des bibliothèques, des établissements scolaires, des associations regroupant des artistes, des associations militantes comme celles pour le droit des femmes ou des habitants. 26 . Le projet pouvait ainsi répondre aux conditions requises par les budgets publics dédiés aux "mémoires de l'immigration ", mais aussi aux fonds destinés aux populations des quartiers dits prioritaires, ce que nous avons découvert au fur et à mesure. 27. "Mémoires des migrations: quelles traces en Région Centre ? ", Journée régionale d'échange organisée par Mémoires plurielles et l'université de Tours, avec le soutien de la DRAC, la DRJSCS et la Région Centre, le 6 décembre 2013, à l'Hôtel de Région, Orléans. 80 personnes étaient présentes et deux articles dans la presse locale ont ensuite restitué le sens de cette journée de travail. 


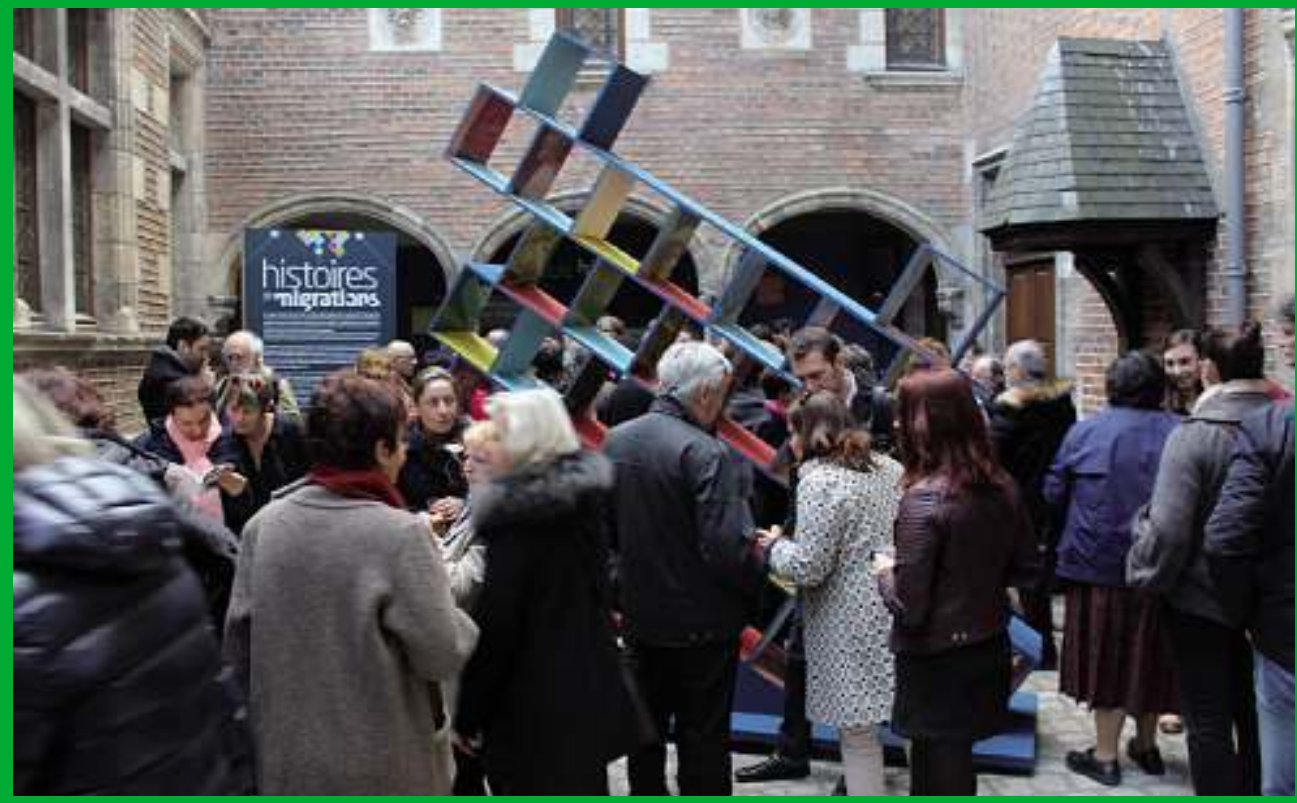

Inauguration de l'exposition Histoires de migrations, à l'Hôtel Cabu, Musée d'histoire et d'archéologie, Orléans, 2017. Photo Pierre Billion-Laroute. (o Pierre Billion-LARoute.

temps, une archiviste était recrutée par l'association afin de repérer et collecter dans la région des documents d'archives pour l'exposition.

\section{Injonction participative ou « démocratie patrimoniale » ?}

Ouvrir la fabrique de l'exposition à ceux susceptibles de discuter ou d'incarner les thématiques traitées est aujourd'hui un principe volontiers affiché par les professionnels des institutions patrimoniales, bien que ce ne soit pas le cas de ceux des musées Orléans. Pour autant, pour les chercheurs comme pour les associations impliquées, cette ouverture devint centrale dans le projet. De cette façon, ils ne seraient pas dominés par la seule perspective scientifique, notamment historique, ni enfermés dans une vision surplombante. Une large participation promettait de "pluraliser " le récit des migrations et de mieux restituer la diversité des expériences. Elle rendrait le propos plus accessible, et éviterait le piège d'un récit "majoritaire » trop souvent prégnant dans ce type d'exposition, celui du "grand récit» de l'intégration ${ }^{28}$. Cette méthode inclusive permettait aussi de «consolider les réseaux associatifs locaux qui ceuvrent dans le sens d'une plus grande reconnaissance des migrations", dans un contexte de "xénophobie montante en Europe $^{29}$ ». 
Si l'idée d'une « démocratie patrimoniale » à l'œuvre fut volontiers avancée au début, elle demeura un principe abstrait, que chacun tenta de s'approprier concrètement, non sans écueil. Au sein des quatre groupes de travail constitués, la réflexion fut souvent happée par la difficulté à penser et mettre en œuvre la « participation ». Dès la première réunion de suivi du projet de recherche, la notion de collaboration a d'ailleurs été proposée comme une alternative plus prudente et moins « piégée $e^{30}$ ». L'analyse à chaud des dynamiques insufflées dans ces groupes de travail ${ }^{31}$ souligne le brouillage que cette démarche a généré lors des premières réunions. Pour les participants, comme pour ceux qui animaient les séances, elle introduisait une incertitude comme si, face à l'injonction participative, les uns et les autres se renvoyaient la responsabilité de l'avancement du projet bien plus qu'ils tentaient de s'approprier un "pouvoir de patrimonialiser ${ }^{32}$ ». Ces observations montrent qu'il ne suffit pas d'inviter des acteurs à participer pour que se déploient des échanges constructifs et symétriques. La " démocratie patrimoniale » se construit dans la reconnaissance des rôles que chacun est prêt à tenir dans l'avancée du projet. Tout s'est donc mis en place peu à peu, confirmant que ce "pouvoir de patrimonialiser " est, d'une part, le résultat d'une pluralisation des instances à l'œuvre autour du patrimoine et, d'autre part, qu'il met davantage en jeu des négociations et des résistances entre acteurs que l'exercice d'une autorité singulière. Mais voyons maintenant les conditions de sa mise en œuvre.

\section{L'enjeu du concernement}

Dans chaque groupe de travail, les participants ont été sollicités au moyen d’appels téléphoniques, mais aussi par le biais de tracts et de courriels envoyés largement aux associations et aux structures sociales et culturelles de la ville et de la presse locale. Au regard d'une diffusion très large de l'invitation à prendre part au projet, force est de constater que les relations et les prises de contacts interpersonnels ont rencontré le meilleur écho, montrant que, comme le souligne à juste titre la sociologue Marion Carrel, la participation «ça se travaille ${ }^{33}$ ». L'interconnaissance préalable opère ainsi dans le sens de l'identification d'une possible affinité entre participants, elle-même favorable à l'engagement à œuvrer ensemble. Une centaine de personnes a finalement contribué à l'exposition régionale, de près ou de loin, de façon directe ou indirecte, par le biais des groupes de travail ou au-delà, dans une relation amicale et/ou professionnelle avec une des personnes y siégeant. Si ce chiffre ne signifie rien au regard de la population régionale, il a néanmoins pour effet d'introduire dans la fabrique de l'exposition une variété de personnes aux profils divers. Si certains sont des militants engagés pour la cause des étrangers et des sans-papiers (qu'ils soient eux-mêmes d'anciens migrants ou non), d'autres sont des professionnels du travail social (animateur socioculturel, écrivain public, éducateur, médiateur culturel), des membres d'associations d'insertion, des professionnels d'organismes d'alphabétisation. D’autres sont artistes (plasticien, comédien, réalisateur, peintre), ou encore porteurs de politiques sociales ou urbaines, élus municipaux, représentants de collectivités locales, enseignants, représentants d'associations de migrants ou de simples "témoins ». Si les profils professionnels croisent souvent des trajectoires de migration, rares sont ceux qui sont venus dans ces groupes au seul titre de la migration. Il n'y a donc pas de lien systématique entre le vécu d'une trajectoire migratoire et l'engagement pour un tel projet: non seulement 
tous ceux qui ont vécu la migration, directement ou indirectement, ne se sentent pas nécessairement concernés par une démarche " patrimoniale », mais elle séduit parfois davantage des personnes qui, pour des raisons diverses (personnelles, professionnelles, politiques), ont été initiées à la «cause migratoire » sans l'avoir eux-mêmes vécue. En revanche, les migrants récemment arrivés sont quasi absents des groupes de travail, la barrière de la langue, les préoccupations administratives et autres impératifs de survie les tenant à distance d'un tel projet.

Lors de ces premières rencontres, les participants ont raconté l'« histoire de migration » qui justifiait leur présence et fait ainsi valoir leur expertise, que ce soit au nom d'un vécu familial, professionnel ou artistique.

Cette absence des uns et cette présence des autres révèlent l'importance, pour une partie des participants, de pouvoir trouver une satisfaction personnelle à sa réalisation; elles soulignent aussi la nécessité que l'engagement pris ici au nom de la mémoire des migrations puisse nourrir une sociabilité qui fasse sens dans la trajectoire de chacun, que celle-ci soit scientifique, artistique, électorale ou associative, ou au sein d'un réseau de relations électives. De ce point de vue, l'engagement repose sur une " prise de pari subsidiaire ${ }^{34}$ », dissociée du projet lui-même, qui tend à mettre en cohérence des actions individuelles. A contrario, la démarche peine à impliquer les personnes isolées ou aux statuts plus incertains et qui peuvent craindre de se voir attribuer une étiquette que leur position rend plus difficile à assumer.

\section{Faire patrimoine avec les concernés}

La réalisation d'une exposition nécessite non seulement d'en décider les contenus et la forme, d'en rassembler les éléments, mais aussi de trouver un lieu et les ressources nécessaires à sa mise en place.
Ces points ont été discutés au sein des groupes de travail au cours d'un processus qui a finalement pris trois ans.

Plusieurs réunions, au début, ont ainsi été occupées à préciser la démarche, à discuter le synopsis, à réfléchir au lieu d'exposition, à s'interroger sur les possibilités de financement. Le binôme d'animateurs de réunion (chargée de mission de l'associationchercheur(e)) a joué un rôle central dans la mise en place d'une dynamique de travail collective. Leurs «casquettes » institutionnelles distinctes masquaient une certaine porosité des postures scientifiques et militantes; les chercheurs assumant de « s'engager » en s'investissant dans ce travail collaboratif, tandis que la chargée de mission de l'association, Pôleth Wadbled, pouvait faire valoir ses compétences scientifiques ${ }^{35}$. Lors de ces premières rencontres, les participants ont raconté l'« histoire de migration » qui justifiait leur présence et fait ainsi valoir leur expertise, que ce soit au nom d'un vécu familial, professionnel ou artistique. La réunion est ainsi devenue le lieu de confrontations d'" expertises du vécu $u^{36}$ » et de savoirs expérientiels, où se réalise parfois la conversion de malaises individuels, vécus jusque-là en silence, en problèmes partagés et publics. Des représentants d'association et des militants y proposent que leur « cause » soit présente dans l'exposition, leur aide pour contacter des personnesressources. Une enseignante de collège, une militante du droit des femmes et un plasticien d'origine marocaine envisagent de construire un projet pédagogique, associatif ou artistique convergent.

Dans ces réunions, la plupart des participants attendent des organisateurs qu'ils prennent le temps d'écouter, d'entendre et de voir ce qui généralement reste inaudible et invisible; qu'ils "creusent » ou approfondissent la recherche à partir de pistes et d'éléments partiels évoqués en réunion; enfin, au bout du processus, qu'ils mettent en cohérence la pluralité des éléments collectés, trouvent les ressources nécessaires et orchestrent sa réalisation. Il a fallu rappeler aux participants le 


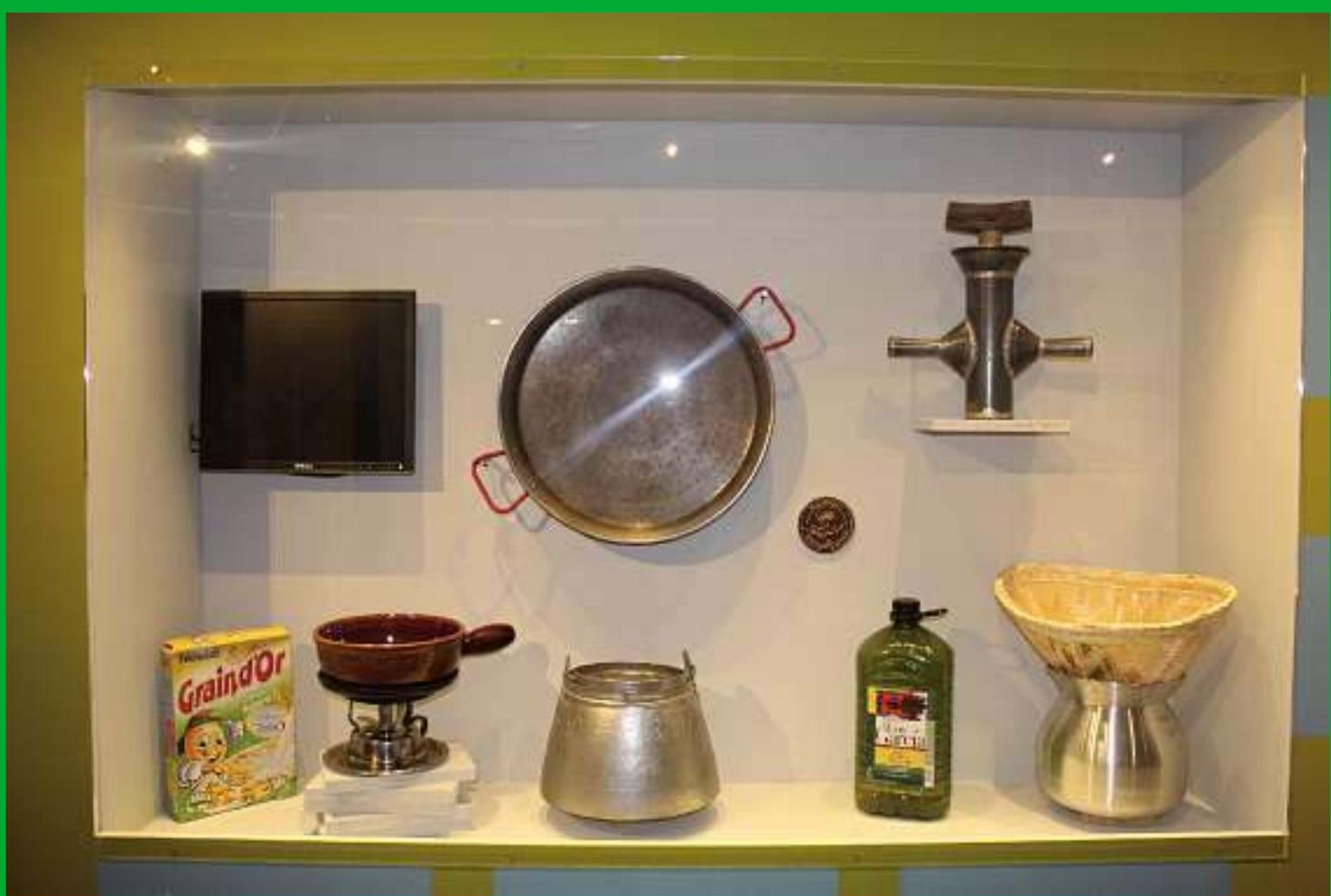

Vitrine des objets culinaires et mémoriels collectés auprès des migrants ou des descendants de migrants en région Centre-Val de Loire. Exposition Histoires de migrations, Hôtel Cabu, Musée d'histoire et d'archéologie, Orléans, 2017. Photo Pierre Billion-Laroute.

(c) Pierre Billion-Laroute.

périmètre régional de la collecte, les tenir informés des dimensions technique et financière du projet, et harmoniser les pratiques et les dynamiques collectives, d'un groupe de travail à l'autre. Au fil des rencontres, la configuration des places et les positionnements de chacun évoluent, révélant des ajustements en situation. Certains envisagent ce dispositif comme une opportunité, le détournant parfois. Le représentant d'un centre social s'en saisit pour le faire entrer dans les objectifs visés par sa structure; un autre modifie finalement son engagement initial pour devenir " porteur d'enjeux », préférant rester en retrait de la collecte. La contribution du centre social qu'il représente est ainsi conditionnée par l'objectif large d'œuvrer au nom "du vivre ensemble ", bordé par de faibles disponibilités de temps. S'il n'y a pas eu de conflits ouverts dans les multiples temps de réunion, des tensions et des oppositions ont toutefois pu s'exprimer dans leurs marges, sous forme de commentaires émis à l'issue de la réunion, auprès de l'un des animateurs, dans l'attente d'un arbitrage de sa part ${ }^{37}$. 
Ils révèlent des rivalités entre les associations impliquées, des attentes inconciliables de certains participants, l'impatience de certains militants, ou la déception de ne pas avoir pu davantage influencer le contenu. Ces attentes n'empêchent pas la concertation, les coopérations et les ajustements pour construire progressivement des démarches convergentes entre le projet régional et les initiatives locales.

La collecte de contenus,

envisagée au début

comme une démarche que chacun pourrait s'approprier, a ainsi donné lieu

à des interprétations variables.
La collecte de contenus, envisagée au début comme une démarche que chacun pourrait s'approprier, a ainsi donné lieu à des interprétations variables. Sans doute faut-il préciser que, pour la plupart des «animateurs » des groupes de travail, la conception de ce que devait être une "collecte » restait relativement vague. Peu d'entre eux en anticipaient les difficultés les contraintes pratiques (assurance, stockage, sélection, documentation). Parmi les participants, quelques rares personnes collectèrent des objets dans leur entourage, voire à leur domicile. Pour beaucoup d'autres, une telle démarche impliquait des compétences dont ils se sentaient dépourvus : savoir identifier et justifier l'intérêt de tel ou tel élément pour une exposition. Alors que l'équipe de coordination craignait une surabondance d'objets que leurs porteurs voudraient tous voir intégrer en bonne place dans l'exposition, ce fut plutôt l'abondance de projets qui s'imposa. Cet emballement des projets suggère, on va le voir, que la cause patrimoniale tend à devenir secondaire, reléguée derrière d'autres enjeux dont la portée mérite d'être interrogée.

\section{De l'engagement patrimonial à la dynamique culturelle}

Les participants aux groupes de travail se sont engagés dans le projet de différentes façons, y jouant des rôles variables. Certains, familiers des rouages politico-administratifs du fait de leur métier, de leurs engagements associatifs ou artistiques, et directement concernés, du fait de leur histoire familiale, par la migration, sont devenus progressivement incontournables: ils ont " épousé » le projet et y ont contribué par leur propre « enquête » qui les a amenés à collecter des objets, des documents, des discours, encouragés par la dynamique du groupe de travail. Ce type d'engagement s'est construit au fil des réunions et révèle une véritable rencontre entre des acteurs aux positions sociales et professionnelles différentes réunies autour du projet. Il mérite d'être souligné car il met en lumière les conditions d'un engagement « patrimonial ». À Tours par exemple, une travailleuse sociale d'origine algérienne a ainsi proposé d'enquêter auprès de familles côtoyées par le biais de son activité professionnelle et de son réseau amical. Cette démarche lui a permis d'acquérir de nouvelles compétences (collaboration avec les chercheurs, posture d'enquête, sélection d'objets), mais aussi d'imposer un choix d'objets à exposer et les « récits de soi » qui les accompagnaient. C'est aussi le cas d'une animatrice socio-culturelle, mariée autrefois à un commerçant marocain et détentrice aujourd'hui d'un master de sociologie, qui s'est appuyée, de la même façon, sur ses réseaux professionnels et personnels pour mener une collecte auprès de femmes du quartier où elle travaille. Toutes deux se sont fortement impliquées tout au long de la démarche, organisant finalement une visite groupée de l'exposition, par le biais de leur mission d'animation socio-culturelle. Pour d'autres, l'engagement pris dans le projet patrimonial ici s'articule avec des effets attendus ailleurs lorsque des conflits agitent la scène politique internationale ${ }^{38}$. La démarche "patrimoniale " prend sens ici dans une démarche personnelle où s'articulent la valorisation des objets collectés et la quête d'une reconnaissance de compétences culturelles.

D'autres, au contraire, n'ont participé qu'à une ou deux séances, pour déposer telle idée ou simplement leur contact, prévenant qu'ils étaient pris par 
d'autres engagements (professionnels ou militants) mais que la thématique ou la cause qu'ils portaient ne devait pas être oubliée ou négligée (la place des femmes, la mémoire des luttes, limportance de la langue ou du travail, les sociabilités sportives, etc.). D'autres enfin ne semblent être venus aux réunions que pour se tenir au courant, tels des "veilleurs ", ne contribuant pas directement eux-mêmes mais souhaitant être informés du devenir d'un tel projet dont les effets pouvaient être importants pour le territoire. Beaucoup d'objets et de documents ont aussi été collectés par les chercheurs, peu de temps avant l'exposition, afin de compléter ce qui leur semblait manquer.

Ce sont finalement environ cinq cents objets et documents ${ }^{39}$ qui ont été rassemblés, couvrant des thématiques très variées. Ces documents et objets, devenus expôts, ont tous été prêtés par leurs propriétaires et rendus après l'exposition. Si le musée a, pendant quelques mois, consacré et valorisé un patrimoine local des migrations, son statut patrimonial au sens classique est resté ponctuel puisque les objets collectés n'ont pas intégré de collection muséale. Une telle patrimonialisation institutionnelle n'a d'ailleurs pas été discutée entre les partenaires du projet, acceptant tous tacitement l'idée d'une valorisation éphémère, sans conservation.

La mise en place de réunions trimestrielles a eu d'autres effets sur la dynamique du projet que nous n'avions pas anticipés. Le fait de se réunir régulièrement a favorisé l'échange d'expériences et la confrontation des points de vue. Sur chaque territoire, les propositions ont fusé dès le début, en particulier sous forme de projets locaux, complémentaires à la grande exposition qui se tiendrait au musée à Orléans, capitale régionale. Les réunions ont donc non seulement permis aux contributeurs de s'approprier la démarche mais ont aussi donné jour à des projets collatéraux. Par exemple, à Montargis, dès la deuxième réunion, plusieurs participants développent l'idée d'une exposition locale qui ne soit "pas une pâle déclinaison" de l'événement régional mais qui propose une thématique propre et complémentaire. Un des artistes présents, lui-même descendant de migrants italiens, évoque alors l'intérêt de présenter la migration de l'intérieur. Ceci amène le groupe à discuter des enjeux de la transmission intergénérationnelle de la langue, de la cuisine et de l'histoire familiale, à partir de l'expérience, du vécu familial ou professionnel de chacun.

Selon les contextes territoriaux, la démarche s'est déployée différemment. À Orléans, par exemple, ces récits d'expérience ont été marqués par l'histoire déjà ancienne d'une mobilisation associative importante, alors que dans les autres agglomérations, où ce type de réseau était moins structuré, les thèmes du travail ou de l'invisibilité des femmes préoccupèrent davantage le groupe. Un autre effet de la collaboration a été l'essaimage du projet dans la ville sous forme de manifestations ou d'événements. Tandis que certains prenaient place dans l'exposition, des œuvres de plasticiens en particulier, la plupart ont motivé une programmation «hors les murs " qui a large-
Ce sont finalement environ cinq cents objets et documents qui ont été rassemblés, couvrant des thématiques très variées. Ces documents et ces objets, devenus expôts, ont tous été prêtés par leurs propriétaires et rendus après l'exposition. ment débordé le projet initial : spectacles vivants, ateliers d'arts plastiques ou créatifs, créations vidéos, projections de film, projets pédagogiques. Ces projets étaient aussi vus comme des outils plus efficaces qu'une patrimonialisation muséale pour œuvrer à la valorisation des migrations. Du point de vue des professionnels (artistes, enseignants, travailleurs sociaux) engagés localement, prendre part au projet régional constitua souvent un horizon d'action qui permettait non seulement de rendre visible et d'élargir la publicisation d'une proposition, mais aussi de faire valoir une aptitude à porter un projet culturel. Ce détournement du projet patrimonial a ainsi contribué à la mise en œuvre d'une dynamique multimodale de valorisation culturelle des migrations. 


\section{Incertitude et complexité d'une dynamique incrémentale}

Nous voudrions terminer par deux remarques: souligner l'incertitude qui a marqué l'ensemble du travail pendant de longs mois et revenir sur la complexité que génère tout travail collaboratif de patrimonialisation.

La première concerne les chercheurs mais aussi l'ensemble des participants: l'incertitude a imprégné de nombreux moments et pratiques tout au long de ces mois de fabrique de l'exposition. L'ensemble du processus collaboratif s'est révélé souvent déstabilisant, sans doute du fait que les étapes et les conditions du travail ne furent pas définies ni contrôlées au départ, mais produites au fur et à mesure, collectivement, dans une dynamique qu'on pourrait qualifier d'incrémentale puisqu'elle s'édifiait progressivement.

La collaboration étroite entre chercheurs, associations et artistes amène nécessairement à repenser les fondements et les frontières de la légitimité des savoirs. Certes, la démarche s'apparente au positionnement inhérent à toute recherche-action qui n'est pas nouveau et rappelle les débats anciens quoique toujours prégnants sur la posture scientifique en tant que telle et ses frontières avec les différentes formes de savoirs. Ici, les postures ont été explicitement hybrides, pour les chercheurs comme pour les autres participants impliqués. L'expérience ne constitua pas une démarche de valorisation scientifique, comme cela avait pu sembler lêtre au départ ; elle est devenue une manière de " faire de la recherche " ensemble, en y associant les interlocuteurs "de terrain ", favorisant une coconstruction du savoir dans une dynamique culturelle collective. L'échange d'expériences et la confrontation des points de vue encouragèrent une acculturation réciproque des " concernés », des militants, des chercheurs, des associations et de professionnels, quills soient du social, de l'urbain, de la culture ou du patrimoine.

La seconde questionne le processus de patrimonialisation sous l'angle de la participation. L'expérience nous a amenés à déplacer notre questionnement du processus de fabrication de la valeur patrimoniale vers celui de l'engagement culturel. On connaît certes de longue date la portée politique du fait patrimonial et les travaux sur les patrimonialisations ont bien décrit la mécanique d'institution de la culture et son effet de légitimation des savoirs, notamment populaires ; ou encore l'importance des "émotions patrimoniales ${ }^{40}$ " lorsque les groupes sociaux se mêlent de «faire » le patrimoine. Cette expérience collective n'a échappé ni à la dimension politique, ni aux émotions partagées, et ce dans un contexte d'interdépendance étroite des acteurs impliqués : de lélu au technicien, du militant au témoin, de l'artiste au scientifique. C'est bien cette palette d'acteurs divers qui dessine la complexité des rouages du "pouvoir de patrimonialiser » autour d'une dynamique réticulaire et incrémentale. 